\title{
NSs protein of severe fever with thrombocytopenia syndrome virus suppresses interferon production through different mechanism than Rift Valley fever virus
}

\author{
S. ZHANG*, B. ZHENG*, T. WANG, A. LI, J. WAN, J. QU, CH. LI, D. LI, M. LIANG
}

Key laboratory of Medical Virology, NHFPC; Department of Viral Hemorrhagic Fever, National Institute for Viral Disease Control and Prevention, China CDC, Beijing 102206, P. R. China

Received July 21, 2016; revised November 28, 2016; accepted June 21, 2017

\begin{abstract}
Summary. - Severe fever with thrombocytopenia syndrome virus (SFTSV) is a newly identified Phlebovirus that causes severe fever with thrombocytopenia syndrome. Our study demonstrated that SFTSV NSs functioned as IFN antagonist mainly by suppressing TBK1/IKKe-IRF3 signaling pathway. NSs interacted with and relocalized TANK-binding kinase 1 (TBK1) into NSs-induced cytoplasmic structures and this interaction could effectively inhibit downstream phosphorylation and dimerization of interferon regulatory factor 3 (IRF3), resulting in the suppression of antiviral signaling and IFN induction. Functional sites of SFTSV NSs binding with TBK1 were then studied and results showed that NSs had lost their IFN-inhibiting activity after deleting the 25 amino acids in N-terminal. Furthermore, the mechanism of Rift Valley fever virus (RVFV) NSs blocking IFN- $\beta$ response were also investigated. Preliminary results showed that RVFV NSs proteins could neither interact nor co-localize with TBK1 in cytoplasm, but suppressed its expression levels, phosphorylation and dimerization of IRF3 in the subsequent steps, resulting in inhibition of the IFN- $\beta$ production. Altogether, our data demonstrated the probable mechanism used by SFTSV to inhibit IFN responses which was different from RVFV and pointed toward a novel mechanism for RVFV suppressing IFN responses.
\end{abstract}

Keywords: SFTS virus; NSs protein; interferon signaling; TBK1/IKKe-IRF3

\section{Introduction}

Severe fever with thrombocytopenia syndrome (SFTS), caused by a novel SFTS virus (SFTSV), is an emerging infectious disease newly identified in China with the fatality rate ranging from $2 \%-15 \%(\mathrm{Li}, 2015)$. As a member of the Phlebovirus species from the family Bunyaviridae, SFTSV is a negative sense, single-stranded RNA virus composed of three segmented genomes. The segments of L, M, and

*Corresponding author. E-mail: mifangl@vip.sina.com; phone: $+86-10-58900822 .{ }^{*}$ These authors contributed equally to this work.

Abbreviations: IFN = interferon; IRF3 = interferon regulatory factor 3; RLU = luciferase units; NSs = nonstructural; NP = nucleoprotein; RIG-I = retinoic acid-inducible gene I; RVFV = Rift Valley fever virus; $\mathrm{SeV}=$ Sendai virus; $\mathrm{SFTS}=$ severe fever with thrombocytopenia syndrome; SFTSV $=$ SFTS virus; TBK1 = TANK-binding kinase 1 ; TLRs $=$ Toll-like receptors
$S$ encode viral RNA polymerase, glycoproteins (Gn and $\mathrm{Gc}$ ), nucleoprotein (NP), and nonstructural (NSs) proteins, respectively. NP and NSs are expressed by separate open reading frames in opposite orientations on $\mathrm{S}$ segment (Yu et al., 2011; Liu et al., 2003). Until now, SFTS has been reported in at least 20 provinces in China and similar disease has also been reported in the United States, South Korea and Japan, showing the risk of disease spread (McMullan et al., 2012; Kim et al., 2013; Takahashi et al., 2013; Feldmann, 2011). SFTS has become a severe threat to public health. However, no antiviral drugs or vaccines are currently available and viral pathogenesis as well as virus-host interactions in humans infected with SFTSV are still largely unknown.

The innate immune response is the first line of host defense against an invading pathogen. A key aspect of the antiviral innate immune response is the synthesis and secretion of type I interferons (IFNs), which exhibit antiviral, anti-proliferative and immunomodulatory functions (Honda et al., 2005; Seth et al., 2006). It is now apparent that multi- 
ple pattern recognition receptor (PRR) families, including Toll-like receptors (TLRs), retinoic acid-inducible gene I (RIG-I)-like receptors (RLRs) and NOD-like receptors (NLRs), significantly contribute to viral detection by sensing viral proteins and nucleic acids, leading to induction of cytokines and IFNs (Kawai and Akira, 2009; Brennan and Bowie, 2010). For RNA virus infection, cytosolic viral RNAs are mainly recognized by RLRs RIG-I and melanoma differentiation-associated protein 5 (MDA5) (Yoneyama and Fujita, 2009), followed by their activation and subsequent translocation to mitochondria where they interact with the common adaptor mitochondrial antiviral signaling protein (MAVS), resulting in the formation of active MAVS polymers (Kawai et al., 2005; Meylan et al., 2005; Hou et al., 2011). MAVS then recruit various signaling proteins such as TNF receptor associated factor 3 (TRAF3) to form antiviral complexes. The TRAF3 binds to MAVS to promote TBK1/ I $\kappa$ B kinase (IKKe)-mediated IRF3 activation (Belgnaoui et al., 2011), and then leads to the production of IFNs. Such response pathways are critical for anti-viral immunity, however they might also trigger autoimmunity response (Hornung and Latz, 2010).

To overcome the antiviral response, viruses have evolved various strategies to inhibit either IFN production, IFN signaling, or IFN action (Garcia-Sastre, 2001; Goodbourn et al., 2000). The efficiency by which a virus antagonizes the IFN system is critical for its pathogenicity and its ability to infect and spread in the host organism. Clinically, IFNs were almost undetectable in the blood of SFTS patients, suggesting that innate immune responses were effectively suppressed. Nonstructural NSs proteins of viruses within the Bunyaviridae family have been found to have variable sizes and coding strategies (Bishop, 1986; Qu et al., 2012). It has been reported that they have some conservative functions, such as regulation of viral replication and IFN antagonism (Schmaljohn et al., 2007). Previous studies showed that NSs proteins of Rift Valley fever virus (RVFV), a virus from the same genus as SFTSV, is a major virulence factor subverting the innate immune defenses of the host (Bouloy et al., 2001; Vialat et al., 2000; Billecocq et al., 2004). As a novel virus, SFTSV NSs proteins might be involved in the suppression of IFN induction, but the mechanism is unclear. Here we show that SFTSV NSs was sufficient for suppression of IFN induction mainly by interfering with the TBK1/IKKe-IRF3 signaling pathway. NSs proteins of SFTSV specifically interact with endogenous intracellular TBK1, followed by inhibition of phosphorylation and dimerization of IRF3, thus suppressing antiviral signaling and IFN induction. Our data also indicate that RVFV NSs inhibits IFN- $\beta$ response by suppressing the expression levels of IRF3. This study demonstrates the probable mechanism used by SFTSV to inhibit IFN production and may point toward a novel mechanism for RVFV suppressing IFN responses, which needs to be further studied.

\section{Materials and Methods}

Cells, viruses and antibodies. Human embryonic kidney 293T (HEK293T) cells (ATCC No. CRL-11268) and African green monkey kidney Vero cells (ATCC No. CCL-8) were cultured in Dulbecco's Modified Eagle Medium (DMEM; Invitrogen) containing $10 \%$ heat-inactivated fetal bovine serum (FBS) and $100 \mathrm{U} / \mathrm{ml}$ penicillin/streptomycin. THP-1 cells (ATCC No. TIB-202) were maintained in RPMI 1640 medium (Invitrogen) containing 10\% FBS and $100 \mathrm{U} / \mathrm{ml}$ penicillin/streptomycin. Cell cultures were incubated at $37^{\circ} \mathrm{C}$ with $5 \% \mathrm{CO}_{2}$. SFTSV strain HB29 (GenBank No. HM745932.1 for S segment, HM745931.1 for M segment, and HM745930.1 for L segment) was used in this study and was propagated in Vero cells in a biosafety level 3 (BSL-3) laboratory. We have used mouse monoclonal antibodies (mAbs) against HA tag (Covance), FITC goat anti-rabbit IgG and CF 555 goat antimouse IgG antibodies which were obtained from Sigma-Aldrich (St. Louis, USA); mouse mAbs against $\beta$-actin, V5 tag antibodies, rabbit mAbs against TBK1, Flag, phospho-TBK1, phospho-IRF3 antibodies and alkaline phosphatase labeled goat anti-rabbit or anti-mouse IgG antibodies were all purchased from Abcam and rabbit polyclonal antibodies against SFTS NSs were produced in our laboratory.

Plasmids construction. Luciferase reporter plasmid for IFN- $\beta$ promoters and mammalian cell expression plasmids for HA-TBK1, Flag-IRF3 were kindly provided by Dr. Dong-Yan Jin (HKU Li Ka Shing Faculty of Medicine). The NP, NSs, Gn, Gc and RdRp protein genes were amplified from the culture supernatants of Vero cells infected with plaque purified SFTSV strain HB29 (passage P2) by RT-PCR and cloned into protein expression vector VR1012 (Hartikka et al., 1996) with or without HA-tag. Expression plasmids for other tagged proteins and NSs mutants were constructed by standard molecular biology techniques.

Dual-luciferase reporter assay. HEK293T cells were seeded in 24-well plates at a density of $2.5 \times 10^{5}$ cells per well one day before they were transfected with NP, NSs, Gn, Gc and RdRp expression plasmids along with pGL3-IFN $\beta$-Luc using FuGENE6 transfection reagent (Roche) according to the manufacturer's instructions. Blanket plasmid VR1012 was added to ensure consistent amount of DNA in each transfection. Twenty-four hours post-transfection, cells were infected with Sendai virus $(\mathrm{SeV})$ for $12 \mathrm{~h}$ and cell lysates were used to determine the Firefly and Renilla luciferase activities using a dual luciferase reporter (DLR) assay kit (Promega) according to the manufacturer's instructions.

Immunoprecipitation. Transfected HEK293T cells were harvested and washed twice with cold PBS, then lysed with lysis buffer (50 mmol/l Tris, pH 7.5, $150 \mathrm{mmol} / \mathrm{l} \mathrm{NaCl}, 1 \mathrm{mmol} / \mathrm{l}$ EDTA, and $0.5 \%$ Triton X-100) supplemented with protease inhibitor cocktail (Roche) and RNase inhibitor (New England BioLabs) on ice for $30 \mathrm{~min}$. Cell lysates were then cleared by centrifugation at 10,000 $\mathrm{x} \mathrm{g}$ for $30 \mathrm{~min}$ at $4^{\circ} \mathrm{C}$ and incubated with anti-HA-agarose beads (Roche) on end-over-end rocker for $4 \mathrm{~h}$ at $4^{\circ} \mathrm{C}$. After five washes with cold lysis buffer, the agarose beads were eluted with $0.1 \mathrm{~mol} / \mathrm{l}$ 
glycine-HCl buffer ( $\mathrm{pH}$ 2.0). The proteins in eluted samples were separated by SDS-PAGE and analyzed by Western blot analysis. Mock- or SFTSV-infected THP-1 cells were lysed in lysis buffer as described above. Cell supernatants pretreated with protein $\mathrm{A} / \mathrm{G}-$ agarose were incubated with anti-NSs antiserum at $4^{\circ} \mathrm{C}$ for $2 \mathrm{~h}$ and then with protein $\mathrm{A} / \mathrm{G}$-agarose at $4^{\circ} \mathrm{C}$ for $4 \mathrm{~h}$. After five washes with the lysis buffer, immunoprecipitates were denatured and subjected to SDS-PAGE and Western blot analysis.

IRF3 dimerization assay. The IRF3 dimerization assay was carried out as described previously (Iwamura et al., 2001). Briefly, transfected cells were resuspended in lysis buffer. The lysates were mixed thoroughly, incubated on ice for $10 \mathrm{~min}$, and then centrifuged at $4^{\circ} \mathrm{C}$ for $5 \mathrm{~min}$ at $10,000 \mathrm{x} \mathrm{g}$. Cleared cell lysates were separated by nondenaturating gel electrophoresis in a $7.5 \%$ native gel. IRF3 monomers and dimers were detected by Western blot analysis using antibodies against IRF3.

Western blot analysis. Transfected cell pellets were lysed in loading buffer $(0.08 \mathrm{~mol} / \mathrm{l}$ Tris with $2 \%$ SDS, $10 \%$ glycerol, $0.1 \mathrm{~mol} / \mathrm{l}$ dithiothreitol, and $0.2 \%$ bromophenol blue, $\mathrm{pH} 6.8$ ) and boiled for $5 \mathrm{~min}$. Proteins were separated by SDS-PAGE or native-PAGE and then transferred to a PVDF membrane (Millipore, Billerica, USA), followed by incubation with corresponding primary antibodies. After four washes, membranes were incubated with alkaline phosphatase- or horseradish peroxidase-conjugated secondary antibodies. After additional washes, BCIP (5-bromo-4-chloro-3indolylphosphate)/ nitroblue tetrazolium or enhanced chemiluminescence reagents (Invitrogen) were used for signal development. The $\beta$-actin levels were detected as an input control.

Immunofluorescence and confocal microscopy. Transfected cells were fixed with $4 \%$ paraformaldehyde (PFA) in PBS at room temperature for $30 \mathrm{~min}$ and were permeabilized with $0.5 \%$ Triton X-100 on ice for $10 \mathrm{~min}$. After three washes with PBS and blocking with $5 \% \mathrm{BSA}$ at $37^{\circ} \mathrm{C}$ for $1 \mathrm{~h}$, cells were incubated with anti-V5 and antiHA antibodies at 1:100 dilution in PBS-Tween (PBST) containing $1 \% \mathrm{BSA}$ at $4^{\circ} \mathrm{C}$ overnight. After three washes with PBST, the cells were incubated with an FITC-conjugated anti-rabbit IgG antibody and CF 555-conjugated anti-mouse IgG antibody at 1:200 dilution at $37^{\circ} \mathrm{C}$ for $1 \mathrm{~h}$. The cells were washed three times with PBST and incubated with $1 \mathrm{~g} / \mathrm{ml}$ DAPI in PBS for $15 \mathrm{~min}$. After three washes with PBST, cells were covered with one drop of anti-fade reagent (Sigma) and observed under confocal microscope (Olympus).

\section{Results}

\section{SFTSV NSs protein suppresses IFN- $\beta$ promoter activation}

HEK293T cells were transfected with IFN- $\beta$ reporter plasmid and then infected by $\mathrm{SeV}$ with/without SFTSV and the effects on activation of IFN- $\beta$ promoter activity was determined by a DLR assay. The results demonstrated that while $\mathrm{SeV}$ infection enhanced the relative luciferase units (RLU) and activation fold of IFN- $\beta$ promoter activity up to
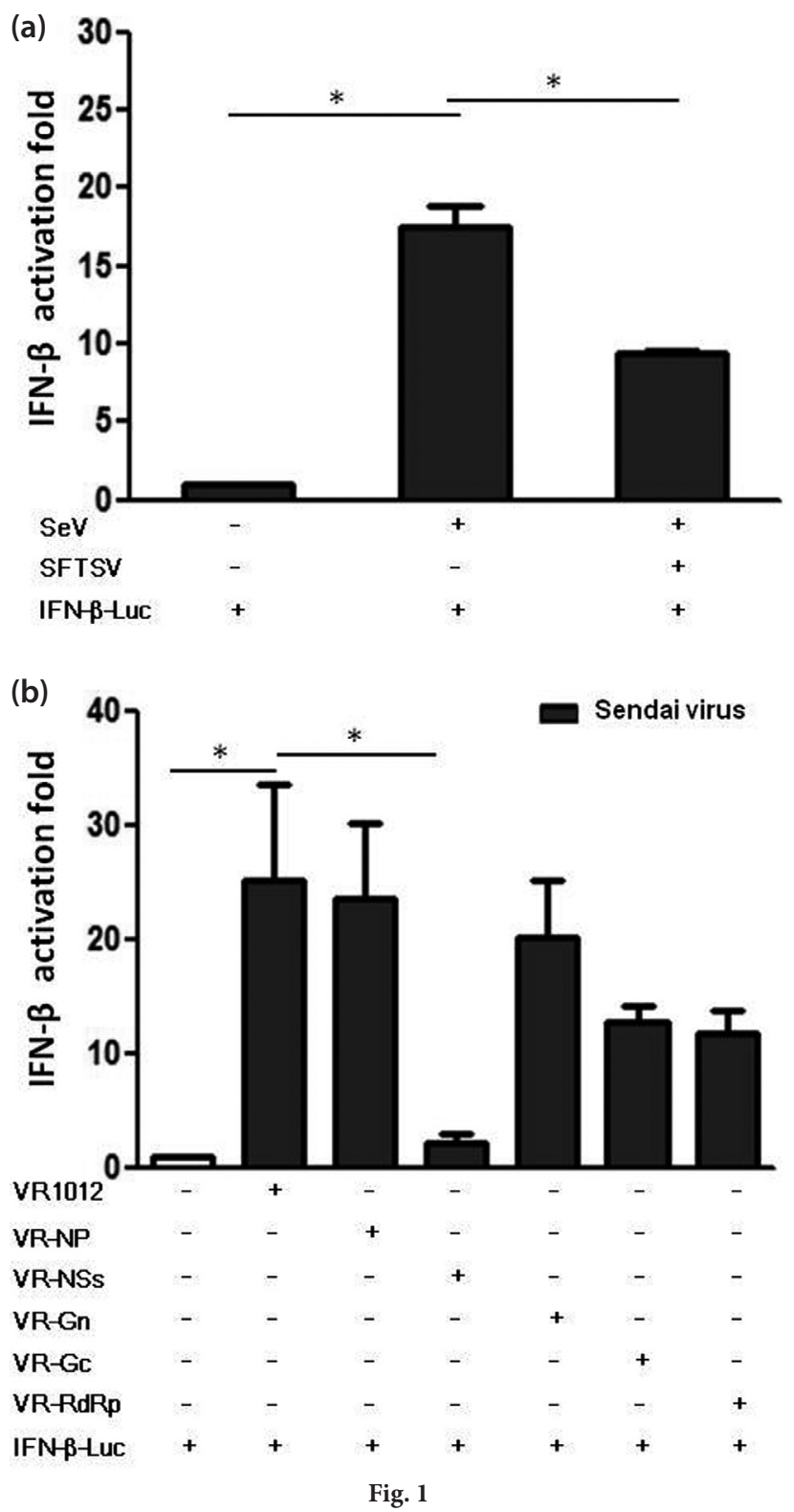

SFTSV NSs suppresses IFN- $\beta$ promoter activity

(a) HEK293T cells were transfected with IFN- $\beta$ reporter plasmid and then stimulated by $\mathrm{SeV}$ with/without SFTSV. (b) HEK293T cells were co-transfected with IFN- $\beta$ reporter plasmid along with plasmids encoding SFTSV NP, NSs, Gn, Gc and L proteins, respectively, followed by $\mathrm{SeV}$ infection. The results of IFN- $\beta$ luciferase activities were described by RLU and activation fold was calculated.

20 -fold, SFTSV reduced IFN- $\beta$ promoter activity down to about $50 \%$ of the original level, indicating that SFTSV suppressed the IFN- $\beta$ promoter activation (Fig. 1a).

As proteins encoded by bunyaviruses, either nonstructural or structural, can act as potential IFN antagonists, a functional screening of the proteins from SFTSV was performed 
(a)

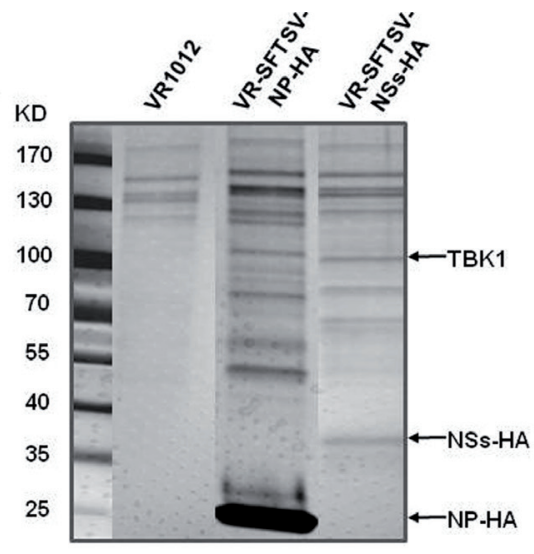

(c)

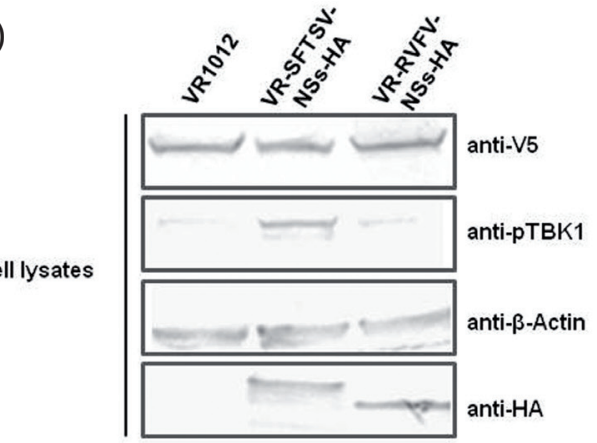

(b)
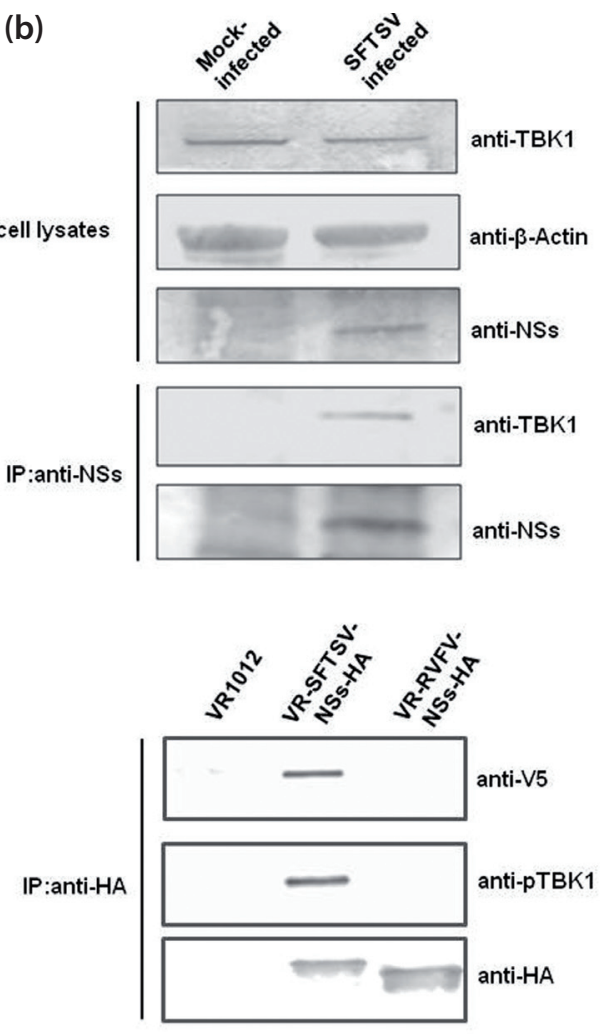

(d)

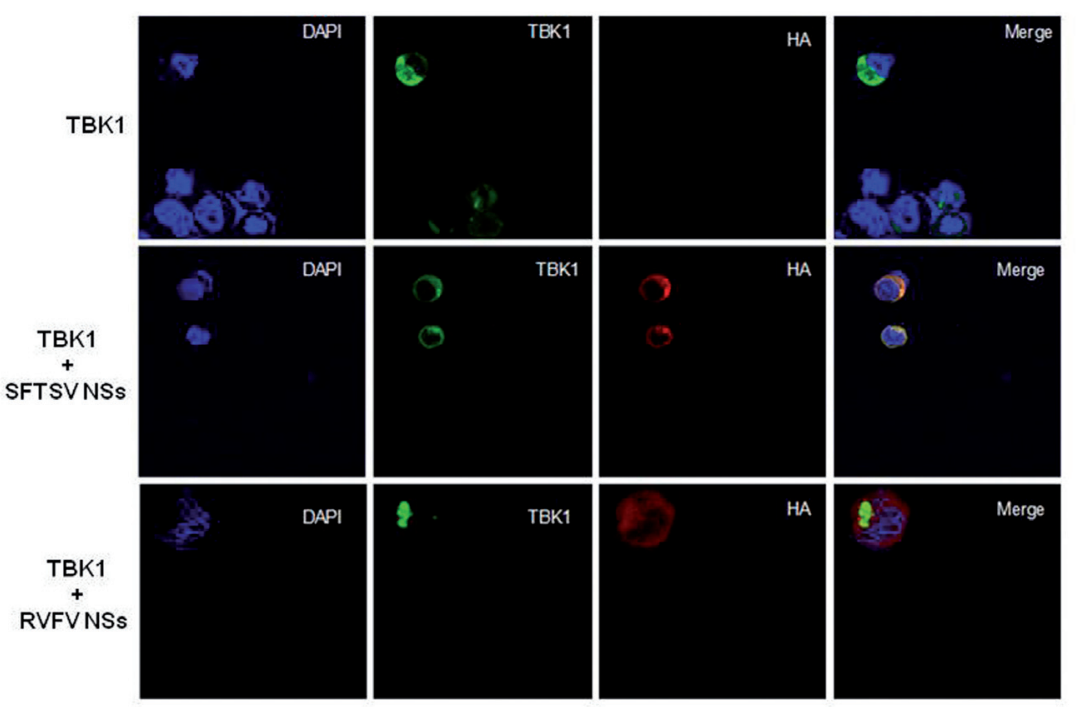

Fig. 2

SFTSV NSs interact with TBK1

(a) HEK293T cells were transfected with blanket VR1012, NP or NSs expression plasmids with HA tag, respectively. 48 h post-transfection, cell lyslates were purified with anti-HA-agarose and subjected to SDS-PAGE. (b) Co-immunoprecipitation of SFTSV and TBK1. Mock- or SFTSV infected THP-1 cell lysates (upper panel) and anti-SFTSV-NSs antibody immunoprecipitated cell lysates (lower panel) were subjected to Western blot analysis using antiTBK1 or anti-NSs antibody. (c) Co-immunoprecipitation of NSs and TBK1. HEK293T cells were transfected with empty VR1012, VR-SFTSV-NSs-HA and VR-RVFV-NSs-HA, together with TBK1 expression plasmid with V5 tag. At $48 \mathrm{~h}$ post-transfection, total cell lysates (left panel) and anti-HA antibody immunoprecipitated cell lysates (right panel) were subjected to Western blot analysis with anti-V5 and anti-phospho-TBK1 antibody. (d) Cellular co-localization of recombinant NSs and TBK1 in 293T cells. Cells were transfected with TBK1 expression plasmid with V5 tag and plasmids encoding HA-tagged SFTSV or RVFV NSs. The localization of proteins after imunofluorescence was visualized by confocal microscopy. 
(Elliott and Weber, 2009). HEK293T cells were cotransfected with IFN- $\beta$ reporter plasmid, along with plasmids encoding the SFTSV NP, NSs, Gn, Gc and L proteins, respectively. Their effects on the activation of IFN- $\beta$ promoter following $\mathrm{SeV}$ infection were examined. Our results showed that NSs protein encoded by the $S$ segment was identified as the main inhibitor of IFN- $\beta$ promoter activation and could significantly reduce IFN- $\beta$ promoter activity down to $20 \%$ of the original level stimulated by $\mathrm{SeV}$. Other proteins including NP which are also encoded by the S segment, Gn and Gc encoded by the $\mathrm{M}$ segment, and L encoded by the L segment, did not reduce IFN- $\beta$ promoter activity as obviously as NSs did (Fig. 1b).

\section{SFTSV NSs interacts with TBK1}

To investigate whether SFTSV NSs protein interacted with any molecules in antiviral signaling pathway, HEK293T cells were transfected with VR1012, VR-SFTS-NP-HA and VR-SFTS-NSs-HA, respectively. Forty-eight hours posttransfection, cleared cells lyslates were incubated with antiHA-agarose and eluted samples were then separated by SDS- (a)

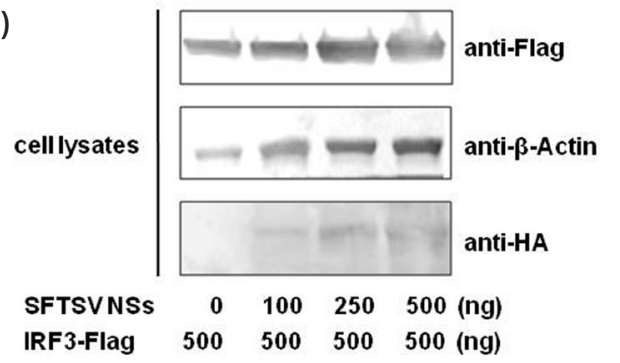

(b)

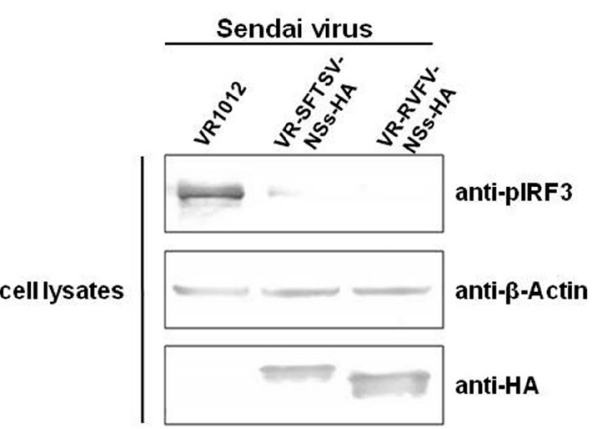

(c)

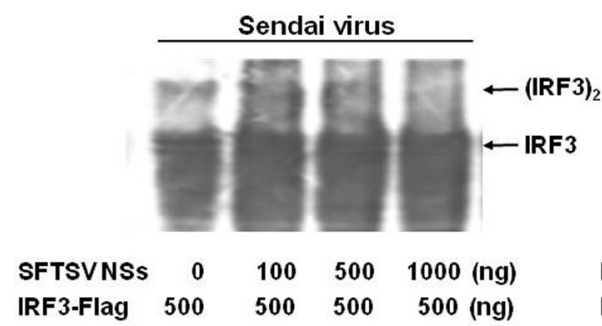

Fig. 3

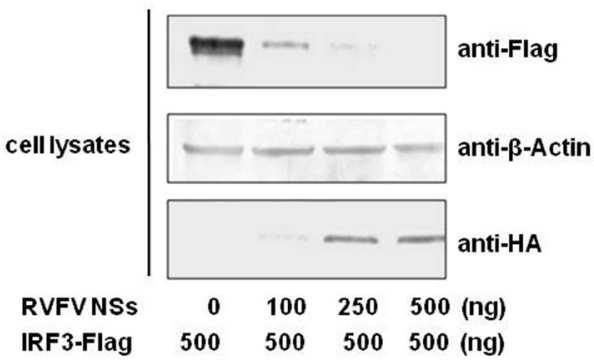

$\begin{array}{lrrrr}\text { IRF3-Flag } & 500 & 500 & 500 & 500 \\ \text { (ng) }\end{array}$

\section{orylation and dimerization of IRF3}

(a) HEK293T cells were transfected with IRF3-Flag expression plasmid together with SFTSV or RVFV NSs expression plasmid with HA-tag in different amounts and the expression levels of IRF3 were evaluated by Western blot analysis. (b) and (c) HEK293T cells were transfected with IRF3-Flag expression plasmid along with the plasmid expressing SFTSV or RVFV NSs-HA tag. Twenty-four hours post-transfection, cells were infected with SeV for $18 \mathrm{~h}$. Cell lysates were separated by SDS- and native-PAGE and analyzed for the phosphorylation (b) or dimerization (c) of IRF3 by Western blot. 
PAGE. A specific band with molecular weight of $\sim 90 \mathrm{kDa}$ was present in VR-SFTS-NSs-HA transfected cells (Fig. 2a) and the results of mass spectrometry identified a critical molecule in the activation of IFN- $\beta$ signaling pathway, TBK1 (GenBank: No.XM_005268810.1).

To examine whether authentic SFTS virus particles interact with TBK1, mock infected or SFTSV infected THP-1 cells were lysed in lysis buffer and incubated with anti-TBK1 and anti-NSs antibodies. Results showed that TBK1 could be detected in both uninfected and infected cells while NSs could only be detected in SFTSV infected cells. Mock- or SFTSV infected THP-1 cells were incubated with anti-NSs antiserum and protein A/Gagarose, followed by Western blot analysis using anti-TBK1 and anti-NSs antibodies, respectively. Our results showed that either
NSs or TBK1 could be detected in SFTSV infected cells, but in uninfected THP-1 cells, neither of these two proteins could be found, indicating that SFTSV could specifically interact with endogenous intracellular TBK1 (Fig. 2b).

The interaction between SFTSV NSs protein and TBK1 was further assessed by immunoprecipitation analysis and immunofluorescent confocal microscopy. HEK293T cells were transfected with VR1012 blanket vector, VR-SFTS-NSsHA and VR-RVFV-NSs-HA, together with TBK1 expression plasmid with V5 tag. The results of immunoprecipitation and Western blot analysis demonstrated that SFTSV NSs proteins did not affect either the expression or the phosphorylation levels of TBK1 (Fig. 2c). For RVFV, NSs protein did not interact with TBK1, suggesting that these two viruses in the

(a)
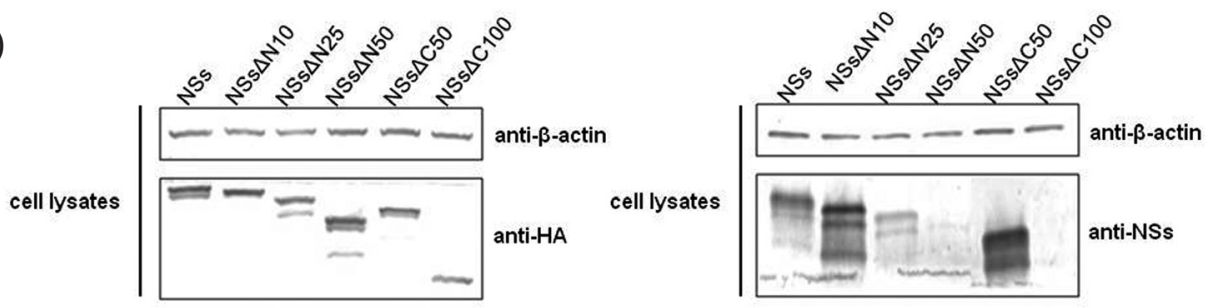

(b)

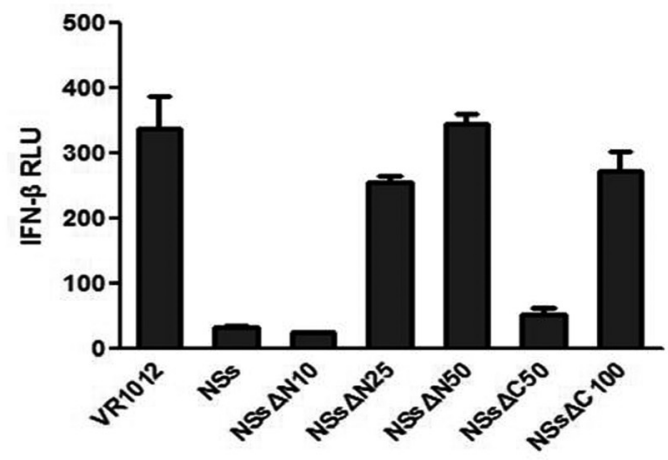

(c)

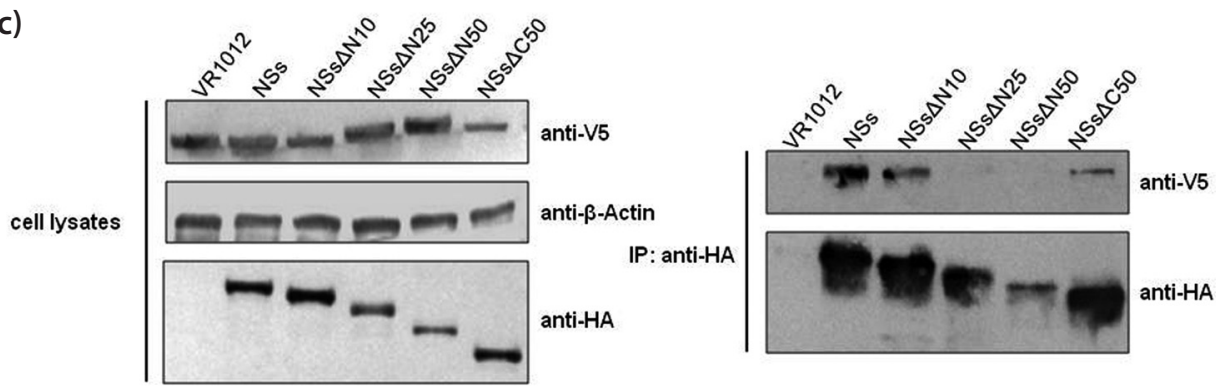

Fig. 4

SFTSV NSs functional sites for binding with TBK1

(a) HEK293T cells were transfected with a series of SFTSV-NSs deletion mutants with HA tag and identified by Western blot analysis using rabbit antiNSs polyclonal antibodies. (b) HEK293T cells were cotransfected with IFN- $\beta$ reporter plasmid along with each SFTSV NSs deletion mutant, followed by SeV stimulation. The results of IFN- $\beta$ luciferase activities were described by RLU. (c) Co-immunoprecipitation of NSs deletion mutants and TBK1. HEK293T cells were transfected with empty VR1012, HA-tagged SFTS NSs or NSs deletion mutants, together with TBK1 expression plasmid with V5 tag, respectively. At $48 \mathrm{~h}$ post-transfection, total cell lysates (left panel) and anti-HA antibody immunoprecipitated cell lysates (right panel) were subjected to Western blot analysis with anti-V5 and anti-HA antibodies. 
genus Phlebovirus might suppress IFN- $\beta$ promoter activation through different mechanisms.

Furthermore, immunofluorescence staining of SFTSV or RVFV NSs and TBK1 was performed to determine their co-localization inside the host cells. TBK1 was diffusely distributed in cytosol in absence of NSs, however, when coexpressed with SFTSV NSs they co-localized in cytoplasm. These results indicate that NSs interact with the kinases TBK1 and relocate to the inclusion bodies. However, cells transfected with RVFV NSs expression plasmid showed morphology changes, nucleus swelling, or even rupture and RVFV NSs protein did not co-localize with TBK1 in cytoplasm, but could be detected in the nucleus (Fig. 2d).

\section{SFTSV NSs inhibits phosphorylation and dimerization of IRF3}

IRF3 is phosphorylated by TBK1, resulting in the dimerization and subsequent nuclear translocation of IRF3 and activates transcription factors during virus infection. Thus, the effects of SFTSV and RVFV NSs on IRF3 were investigated. Firstly, the expression level of IRF3 was evaluated by Western blot analysis. HEK293T cells were transfected with IRF3-Flag expression plasmid along with VR-SFTSV-NSsHA or VR-RVFV-NSs-HA in different amounts. The results showed that levels of IRF3 were not obviously affected when co-transfected with SFTSV NSs expression plasmid whereas RVFV NSs protein could suppress the expression levels of IRF3, in a dose dependent manner (Fig. 3a).

Further, levels of IRF3 phosphorylation were also evaluated by Western blot analysis. SeV infection noticeably triggered IRF3 phosphorylation, which however, was significantly inhibited by SFTSV and RVFV NSs expression (Fig. 3b). The effect of NSs on IRF3 dimerization was analyzed by native-PAGE. As shown in Figure $3 c, \mathrm{SeV}$-induced dimerization of IRF3 was reduced significantly by the expression of SFTSV NSs showing a dose dependent manner. As for RVFV, NSs could reduce the $\mathrm{SeV}$-induced dimerization of IRF3, due to the suppression of IRF3 monomer levels.

\section{SFTSV NSs functional sites for binding with TBK1}

To study the functional sites of SFTSV NSs, a series of NSs deletion mutants with HA tag were constructed and identified by Western blot analysis. After transfection into HEK293T cells, deletion mutants NSs $\Delta \mathrm{N} 10, \mathrm{NSs} \Delta \mathrm{N} 25$ and NSs $\Delta \mathrm{C} 50$, like wild type NSs protein, could be detected by rabbit antiNSs antibodies whereas NSs $\Delta$ N50 and NSs $\Delta$ C100 could not be detected (Fig. 4a), suggesting antigenic changes after deleting the $50 \mathrm{~N}$-terminal amino acids and $100 \mathrm{C}$-terminal

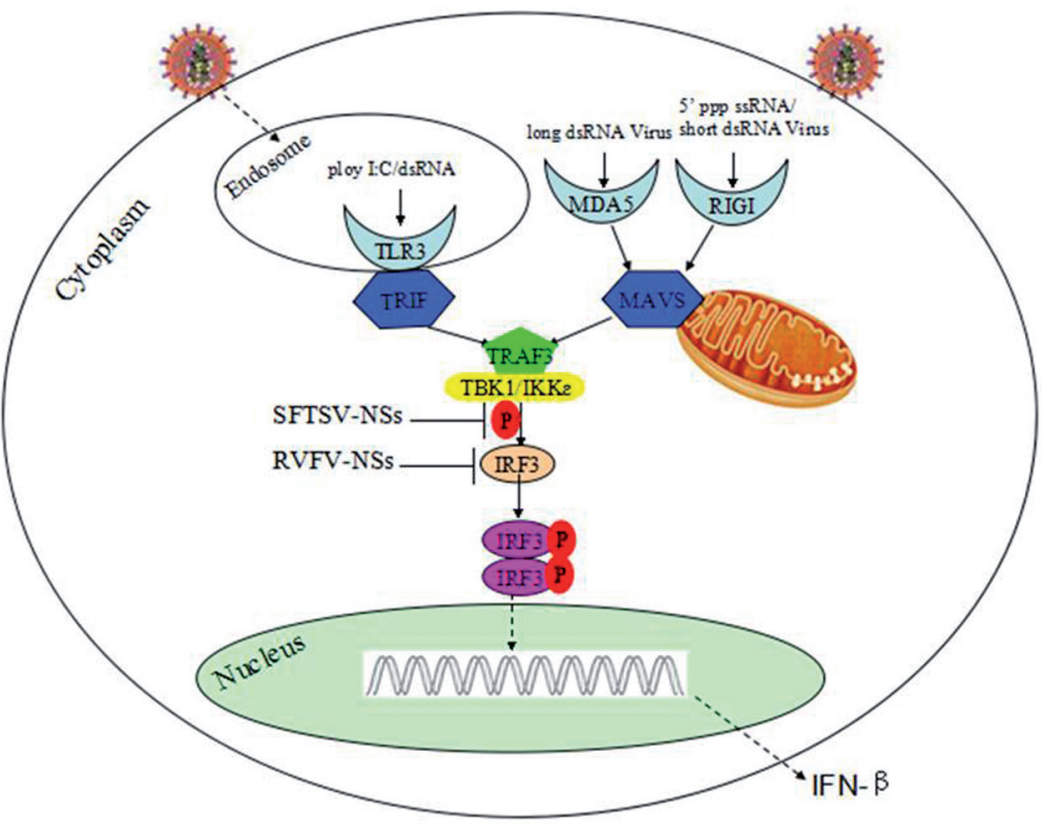

Fig. 5

Pathways analysis of antiviral IFN response in SFTSV and RVFV infected cells

Recognition of RNA viral pathogens by TLRs or RLRs results in the activation of type I interferon (IFN) response. SFTSV nonstructural protein NSs interacts with and relocalizes TBK1 into NSs-induced cytoplasmic structures, then inhibits downstream phosphorylation and dimerization of IRF3, resulting in the suppression of antiviral signaling and IFN induction. RVFV-NSs inhibits the production of IFN- $\beta$ mainly by suppressing the expression and then phosphorylation and dimerization of IRF3. 
amino acids of SFTSV NSs protein. Further, each NSs deletion mutant was transfected into HEK293T cells together with IFN- $\beta$ reporter plasmid. SeV infection noticeably enhanced the RLU of IFN- $\beta$ promoter activity, NSs $\Delta$ N10 and NSs $\Delta$ C50 could significantly reduce IFN- $\beta$ promoter activity down to $20 \%$ of the original level just as NSs protein while activity of IFN- $\beta$ promoter could not be reduced by deletion mutants NSs $\Delta$ N25, NSs $\Delta$ N50 and NSs $\Delta$ C100 (Fig. 4b).

To investigate the interaction between NSs deletion mutants and TBK1, cells were co-transfected with each deletion mutant and TBK1 expression plasmid with V5 tag and then detected by immunoprecipitation and Western blot analysis as described above. Our results demonstrated that any NSs deletion mutants did not affect the expression level of TBK1, however NSs $\Delta$ N10 and NSs $\triangle$ C50 could interact with TBK1 specifically. When deleting the $25 \mathrm{~N}$-terminal mino acids, the interaction between SFTSV NSs and TBK1 was not observed (Fig. 4c).

\section{Discussion}

As a newly emerging infectious disease, SFTS is a serious public health concern. However, details about the viral pathogenesis as well as virus-host interactions are still unclear. Recognition of viral pathogens by TLRs, RLRs or NLRs results in the activation of IFNs response. IFN- $\beta$ has been implicated in many human diseases related to inflammation and recent clinical and laboratory findings have shed new light on the roles of IFN- $\beta$ in human health and disease. IFN- $\beta$ exerts a wide range of biological activities in the human immune system, including the initiation of antiviral protein synthesis, promotion of cytotoxic activity, and driving the differentiation and maturation of certain leukocytes (Vialat et al., 2000; Ito et al., 2001; Kito et al., 2002; Longhi et al., 2009; Nguyen et al., 2002; Su and David, 1999). Viruses, in turn, were found to interfere with induction of IFN synthesis, IFN-induced signaling events, the antiviral effector proteins, or simply shut-off of the host cell macromolecule synthesis machinery to avoid booting of the antiviral host defense. For instance, the influenza A virus NS1 protein prevents IRF3 activation and subsequent IFN production by binding to and sequestering dsRNA (Garcia-Sastre, 2001; Talon et al., 2000); the paramyxoviral V protein as well as the rabies virus $\mathrm{P}$ protein have a dual anti-IFN function as they block both IFN induction (Andrejeva et al., 2004; Childs et al., 2007; Brzozka et al., 2005) and STAT signaling (Andrejeva et al., 2002; Didcock et al., 1999; Brzozka et al., 2006).

The nonstructural NSs protein of phleboviruses has been reported to be a major virulence factor subverting the innate immune defenses of the host and adopted different strategies to counteract the innate immune system. For example, RVFV NSs associate with the cellular partner Sin3A-associated protein 30 (SAP30) and YY1 proteins to inhibit IFN- $\beta$ gene transcription and phosphorylation of eukaryotic initiation factor 2 alpha (eIF2) by posttranslational downregulation of the dsRNA-dependent protein kinase $\mathrm{R}$ (PKR) through the proteasomal pathway, and induce a general transcriptional shut-off through proteasomal degradation of the p62 subunit of the basal transcription factor TFIIH. Toscana virus (TOSV) NSs counteract subsequent IFN gene transcription by specific degradation of PKR and RIG-I in infected cells. As for SFTSV, a deadly bunyavirus newly identified in China, the mechanism of NSs suppressing IFN production was not fully identified. In this study, we analyzed SFTSV and RVFV NSs proteins with the ability to inhibit type I IFN responses and sought to describe the mechanism. Consistent with previous reporting, we found that the SFTSV NSs functions as IFN antagonist mainly by suppressing TBK1/IKKe-IRF3 signaling pathway. NSs interact with and relocalize TBK1 into NSs-induced cytoplasmic structures, and this interaction effectively inhibits downstream IRF3 phosphorylation and dimerization, thus suppresses antiviral signaling and IFN induction (Fig. 5). Furthermore, functional sites of SFTSV NSs associated with TBK1 were then studied. Results showed that NSs had lost their TBK1-binding and IFN-inhibiting activities after deleting the $25 \mathrm{~N}$-terminal amino acids and $100 \mathrm{C}$-terminal amino acids but hadn't after deleting $10 \mathrm{~N}$ terminal amino acids and $50 \mathrm{C}$-terminal amino acids. This result suggests that $10-25 \mathrm{~N}$-terminal amino acids and 50-100 C-terminal amino acids of SFTSV NSs are required for TBK1 association and subsequent IFN inhibition.

In contrast, the mechanism of RVFV NSs blocking IFN- $\beta$ response was different from that of SFTSV. The preliminary result of our study showed that RVFV NSs protein could neither interact with nor co-localize with TBK1 in cytoplasm, but could suppress the expression levels and then phosphorylation and dimerization of IRF3 in the subsequent steps and finally inhibit the production of IFN- $\beta$ (Fig. 5). However, our findings are inconsistent with previous studies which showed that RVFV NSs do not affect IRF3 activation and do not prevent the activation of IFN-specific transcription factors but act at a subsequent step. Thus, further work needs to be done to explain the mechanism of RVFV NSs inhibiting IFN production.

Acknowledgements. This work was supported by Natural Science Foundation of China-Hong Kong Research Grants Council Joint Research Scheme: and the National Key Research and Development Program of China (2016YFC1200200).

\section{References}

Andrejeva J, Childs KS, Young DF, Carlos TS, Stock N, Goodbourn S, Randall RE (2004): The V proteins of paramyxoviruses bind the IFN-inducible RNA helicase, mda-5, and inhibit its activation of the IFN-beta promoter, Proc. Natl. Acad. 
Sci. USA 101, 17264-17269. https://doi.org/10.1073/ pnas.0407639101

Andrejeva J, Young DF, Goodbourn S, Randall RE (2002): Degradation of STAT1 and STAT2 by the V proteins of simian virus 5 and human parainfluenza virus type 2, respectively: consequences for virus replication in the presence of alpha/beta and gamma interferons, J. Virol. 76, 2159-2167. https://doi.org/10.1128/jvi.76.5.2159-2167.2002

Belgnaoui SM, Paz S and Hiscott J (2011): Orchestrating the interferon antiviral response through the mitochondrial antiviral signaling (MAVS) adapter. Curr. Opin. Immunol. 23, 564-572. https://doi.org/10.1016/j.coi.2011.08.001

Billecocq A, Spiegel M, Vialat P, Kohl A, Weber F, Bouloy M, Haller O (2004): NSs protein of Rift valley fever virus blocks interferon production by inhibiting host gene transcription. J. Virol. 78, 9798-9806. https://doi.org/10.1128/ IVI.78.18.9798-9806.2004

Bishop DH (1986): Ambisense RNA genomes of arenaviruses and phleboviruses. Adv. Virus Res. 31, 1-51. https://doi. org/10.1016/S0065-3527(08)60261-4

Bishop DH (1986): Ambisense RNA viruses: positive and negative polarities combined in RNA virus genomes. Microbiol. Sci. 3, 183-187.

Bouloy M, Janzen C, Vialat P, Khun H, Pavlovic J, Huerre M, Haller $\mathrm{O}$ (2001): Genetic evidence for an interferon-antagonistic function of Rift Valley fever virus nonstructural protein NSs. J. Virol. 75, 1371-1377. https://doi.org/10.1128/ JVI.75.3.1371-1377.2001

Brennan K, Bowie AG (2010): Activation of host pattern recognition receptors by viruses. Curr. Opin. Microbiol. 13, 503-507. https://doi.org/10.1016/j.mib.2010.05.007

Brzozka K, Finke S, Conzelmann KK (2006): Inhibition of interferon signaling by rabies virus phosphoprotein $\mathrm{P}$ : activation-dependent binding of STAT1 and STAT2, J. Virol. 80, 2675-2683. https://doi.org/10.1128/JVI.80.6.2675$\underline{2683.2006}$

Brzozka K, Finke S, Conzelmann KK (2005): Identification of the rabies virus alpha/beta interferon antagonist: phosphoprotein $\mathrm{P}$ interferes with phosphorylation of interferon regulatory factor 3, J. Virol. 79, 7673-7681. https://doi. org/10.1128/JVI.79.12.7673-7681.2005

Childs K, Stock N, Ross C, Andrejeva J, Hilton L, Skinner M, Randall R, Goodbourn S (2007): mda-5, but not RIG-I, is a common target for paramyxovirus $\mathrm{V}$ proteins, Virology 359, 190-200. https://doi.org/10.1016/j.virol.2006.09.023

Didcock L, Young DF, Goodbourn S, Randall RE (1999): The $\mathrm{V}$ protein of simian virus 5 inhibits interferon signalling by targeting STAT1 for proteasome-mediated degradation, J. Virol. 73, 9928-9933.

Elliott RM, Weber F (2009): Bunyaviruses and the type I interferon system. Viruses 1003-1021. https://doi.org/10.3390/ $\underline{\mathrm{v} 1031003}$

Feldmann H (2011): Truly emerging - a new disease caused by a novel virus. New Eng. J. Med. 364, 1561-1563. https:// doi.org/10.1056/NEJMe1102671

Garcia-Sastre A (2001): Inhibition of interferon-mediated antiviral responses by influenza A viruses and other negative- strand RNA viruses. Virology 279, 375-384. https://doi. org/10.1006/viro.2000.0756

Goodbourn S, Didcock L, Randall RE (2000): Interferons: cell signalling, immune modulation, antiviral response and virus countermeasures. J. Gen. Virol. 81, 2341-2364. https://doi.org/10.1099/0022-1317-81-10-2341

Hartikka J, Sawdey M, Cornefert-Jensen F, Margalith M, Barnhart K, Nolasco M, Vahlsing HL, Meek J, Marquet M, Hobart P, Norman J, Manthorpe M (1996): An improved plasmid DNA expression vector for direct injection into skeletal muscle. Hum. Gene Ther. 7, 1205-1217. https://doi. org/10.1089/hum.1996.7.10-1205

Honda K, Yanai H, Takaoka A, Taniguchi T (2005): Regulation of the type I IFN induction: a current view. Int. Immunol. 17, 1367-1378. https://doi.org/10.1093/intimm/dxh318

Hornung V, Latz E (2010): Intracellular DNA recognition. Nat. Rev. Immunol. 10, 123-130. https://doi.org/10.1038/nri2690

Hou F, Sun L, Zheng H, Skaug B, Jiang QX, Chen ZJ (2011): MAVS forms functional prion-like aggregates to activate and propagate antiviral innate immune response. Cell 2011, 146, 448-461. https://doi.org/10.1016/j.cell.2011.06.041

Ito T, Amakawa R, Inaba M, Ikehara S, Inaba K, Fukuhara S (2001): Differential regulation of human blood dendritic cell subsets by IFNs. J. Immunol. 166, 2961-2969. https://doi. org/10.4049/jimmunol.166.5.2961

Iwamura T, Yoneyama M, Yamaguchi K, Suhara W, Mori W, Shiota K, Okabe Y, Namiki H, Fujita T (2001): Induction of IRF$3 /-7$ kinase and NF- $\kappa B$ in response to double-stranded RNA and virus infection: common and unique pathways. Genes Cells 6, 375-388. https://doi.org/10.1046/j.13652443.2001.00426.x

Kawai T, Akira S (2009): The roles of TLRs, RLRs and NLRs in pathogen recognition. Int. Immunol. 21, 317-337. https:// doi.org/10.1093/intimm/dxp017

Kawai T, Takahashi K, Sato S, Coban C, Kumar H, Kato H, Ishii KJ, Takeuchi O, Akira S (2005): IPS-1, an adaptor triggering RIG-I- and Mda5-mediated type I interferon induction. Nat. Immunol. 6, 981-988. https://doi.org/10.1038/ $\underline{\text { ni1243 }}$

Kim KH, Yi J, Kim G, Choi SJ, Jun KI, Kim NH, Choe PG, Kim NJ, Lee JK, Oh MD (2013): Severe fever with thrombocytopenia syndrome, South Korea, 2012. Emerg. Infect. Dis. 19, 1892. https://doi.org/10.3201/eid1911.130792

Kito T, Kuroda E, Yokota A, Yamashita U (2002): Enhancement of macrophage cytotoxicity against murine gliomas by interferon beta: increase in nitric oxide production in response to gliomaderived soluble factors. J. Neurosurg. 97, 619-626. https://doi.org/10.3171/jns.2002.97.3.0619

Li D (2015): Severe fever with thrombocytopenia syndrome: A newly discovered emerging infectious disease. Clin. Microbiol. Infect. 21, 614-620. https://doi.org/10.1016/j. cmi.2015.03.001

Liu DY, Tesh RB, Travassos Da Rosa AP, Peters CJ, Yang Z, Guzman H, Xiao SY (2003): Phylogenetic relationships among members of the genus Phlebovirus (Bunyaviridae) based on partial M segment sequence analyses. J. Gen. Virol. 84, 465-473. https://doi.org/10.1099/vir.0.18765-0 
Longhi MP, Trumpfheller C, Idoyaga J, Caskey M, Matos I, Kluger C, Salazar AM, Colonna M, Steinman RM (2009): Dendritic cells require a systemic type I interferon response to mature and induce CD4? Th1 immunity with poly IC as adjuvant. J. Exp. Med. 206, 1589-1602. https://doi. org/10.1084/jem.20090247

Meylan E, Curran J, Hofmann K, Moradpour D, Binder M, Bartenschlager R, Tschopp J (2005): Cardif is an adaptor protein in the RIG-I antiviral pathway and is targeted by hepatitis C virus. Nature $437,1167-1172$. https://doi. org/10.1038/nature04193

McMullan LK, Folk SM, Kelly AJ, MacNeil A, Goldsmith CS, Metcalfe MG, Batten BC, Albari-o CG, Zaki SR, Rollin PE, Nicholson WL, Nichol ST (2012): A new phlebovirus associated with severe febrile illness in Missouri. New Eng. J. Med. 367, 834-841. https://doi.org/10.1056/NEJMoa1203378

Nguyen KB, Salazar-Mather TP, Dalod MY, Van Deusen JB, Wei XQ, Liew FY, Caligiuri MA, Durbin JE, Biron CA (2002): Coordinated and distinct roles for IFN-alpha beta, IL-12, and IL-15 regulation of NK cell responses to viral infection. J. Immunol.169, 4279-4287. https://doi.org/10.4049/ ¡immunol.169.8.4279

Qu B, Qi X, Wu X, Liang M, Li C, Cardona CJ, Xu W, Tang F, Li Z, Wu B, Powell K, Wegner M, Li D, Xing Z (2012): Suppression of the interferon and NF-kappa $B$ responses by severe fever with thrombocytopenia syndrome virus. J. Virol. 86, 8388-8401. https://doi.org/10.1128/JVI.00612-12

Schmaljohn CS, Nichol ST (2007): Bunyaviridae. In Knipe DM and Howley PM (Eds): Fields Virology. Philadelphia : Lippincott, Williams \& Wilkins, pp. 1741-1789.

Seth RB, Sun L, Chen ZJ (2006): Antiviral innate immunity pathways. Cell Res. 16, 141-147 https://doi.org/10.1038/ sj.cr.7310019

Su L, David M (1999): Inhibition of B cell receptor-mediated apoptosis by IFN. J. Immunol. 162, 6317-6321.

Takahashi T, Maeda K, Suzuki T, Ishido A, Shigeoka T, Tominaga T, Kamei T, Honda M, Ninomiya D, Sakai T, Senba T,
Kaneyuki S, Sakaguchi S, Satoh A, Hosokawa T, Kawabe Y, Kurihara S, Izumikawa K, Kohno S, Azuma T, Suemori K, Yasukawa M, Mizutani T, Omatsu T, Katayama Y, Miyahara M, Ijuin M, Doi K, Okuda M, Umeki K, Saito T, Fukushima K, Nakajima K, Yoshikawa T, Tani H, Fukushi S, Fukuma A, Ogata M, Shimojima M, Nakajima N, Nagata N, Katano H, Fukumoto H, Sato Y, Hasegawa H, Yamagishi T, Oishi K, Kurane I, Morikawa S, Saijo M (2014): The first identification and retrospective study of severe fever with thrombocytopenia syndrome in Japan. J. Infect. Dis. 209, 816-827. https://doi.org/10.1093/ infdis/jit603

Talon J, Horvath CM, Polley R, Basler CF, Muster T, Palese P, García-Sastre A (2000): Activation of interferon regulatory factor 3 is inhibited by the influenza A virus NS1 protein. J. Virol. 74, 7989-7996. https://doi.org/10.1128/ IVI.74.17.7989-7996.2000

Vialat P, Billecocq A, Kohl A, Bouloy M (2000): The S segment of Rift Valley fever phlebovirus (Bunyaviridae) carries determinants for attenuation and virulence in mice. J. Virol. 74, 1538-1543. https://doi.org/10.1128/JVI.74.3.1538$\underline{1543.2000}$

Yoneyama M, Fujita T (2009): RNA recognition and signal transduction by RIG-I like receptors. Immunol. Rev. 227, 54-65. https://doi.org/10.1111/j.1600-065$\underline{X .2008 .00727 . x}$

Yu XJ1, Liang MF, Zhang SY, Liu Y, Li JD, Sun YL, Zhang L, Zhang QF, Popov VL, Li C, Qu J, Li Q, Zhang YP, Hai R, Wu W, Wang Q, Zhan FX, Wang XJ, Kan B, Wang SW, Wan KL, Jing HQ, Lu JX, Yin WW, Zhou H, Guan XH, Liu JF, Bi ZQ, Liu GH, Ren J, Wang H, Zhao Z, Song JD, He JR, Wan T, Zhang JS, Fu XP, Sun LN, Dong XP, Feng ZJ, Yang WZ, Hong T, Zhang Y, Walker DH, Wang Y, Li DX (2011): Fever with thrombocytopenia associated with a novel bunyavirus in China. New Engl. J. Med. 364, 1523-1532. https://doi.org/10.1056/ $\underline{\text { NEJMoa1010095 }}$ 